\title{
Análisis de las inteligencias múltiples y creatividad en universitarios
}

\author{
Analysis of multiple intelligences and creativity in university students \\ Sergio Hidalgo Fuentes ${ }^{1}$ \\ María Josefa Sospedra-Baeza ${ }^{2}$ \\ Isabel Martínez-Álvarez ${ }^{3}$ \\ ${ }^{1}$ Facultad de Psicología, Universitat de València. España
${ }^{2}$ Facultad de Filosofía y Ciencias de la Educación, Universitat de València. España
${ }^{3}$ Facultad de Ciencias de la Salud y Educación, Universidad a Distancia de Madrid.España
}

Resumen: Un reto de la educación actual es formar a alumnos competentes para desenvolverse en una sociedad diversa y dinámica. El cociente intelectual no es suficiente para garantizar el éxito académico ni profesional (Extremera \& Fernández-Berrocal, 2006) y deben considerarse aspectos cognitivos, sociales y emocionales. El objetivo del estudio fue analizar el grado alcanzado en estudiantes universitarios de trabajo social en las inteligencias múltiples, inteligencia emocional, creatividad y rendimiento académico, así como su relación y las diferencias en función del sexo y el curso en una muestra de 100 estudiantes. Se encontraron diferencias significativas en función del curso y relaciones entre algunos componentes emocionales e inteligencias múltiples. Así, los resultados hallados ponen de manifiesto la relevancia y relación entre las variables evaluadas para el rendimiento global de los estudiantes universitarios y, por tanto, la pertinencia y necesidad de diseñar intervenciones dirigidas a la optimización de las mismas de manera integrada.

Palabras clave: inteligencias múltiples, inteligencia emocional, creatividad, rendimiento académico, estudiantes universitarios

\begin{abstract}
A challenge of current education is to train competent students to function in a diverse and dynamic society. IQ is not enough to guarantee academic or professional success (Extremera \& Fernández-Berrocal, 2006) and must be considered cognitive, social and emotional aspects. The aim of the study was to analyze the degree attained in university students of social work in multiple intelligences, emotional intelligence, creativity and academic performance, as well as their relationship and differences according to sex and the course in a sample of 100 students. Significant differences were found according to the course and relationships between some emotional components and multiple intelligences. Thus, the results found reveal the relevance and relationship between the variables evaluated for the overall performance of university students and therefore, the relevance and need to design interventions aimed at optimizing them in an integrated manner.
\end{abstract}

Keywords: multiple intelligences, emotional intelligence, creativity, academic achievement, university students

Cómo citar este artículo:

Hidalgo, S. F., Sospedra-Baeza, M. J., \& Martínez-Álvarez, I. (2018). Análisis de las inteligencias múltiples y creatividad en universitarios. Ciencias Psicológicas, 12(2), 271-281.

doi: https://doi.org/10.22235/cp.v12i2.1691

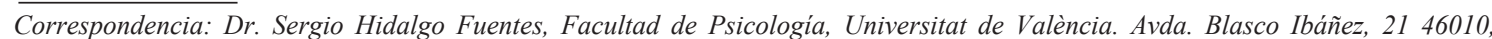
Valencia; e-mail: sergio.hidalgo@uv.es. Dra. María Josefa Sospedra-Baeza, Facultad de Filosofía y Ciencias de la Educación, Universitat de València. Avda. Blasco Ibáñez, 21 46010,Valencia; e-mail: Maria.Jose.Sospedra@uv.es. Dra. Isabel Martínez-Álvarez, Facultad de Ciencias de la Salud y Educación - Universidad a Distancia de Madrid (UDIMA). Carretera de A Coruña, km. 38,5. Vía de servicio,no15.28400, Collado Villalba, Madrid; e-mail: isabel.martinez.al@udima.es 


\section{Introducción}

Hoy en día, en el campo de la psicología educativa, uno de los principales desafíos es el de formar a personas competentes para desenvolverse en la incierta y cambiante sociedad en la que vivimos. De ahí que los profesionales educativos se cuestionen cómo pueden enseñar a sus alumnos a aprender a aprender para que alcancen esta meta de manera autónoma y eficaz (Martín $\&$ Moreno, 2008). Desde hace décadas, existe un acuerdo prácticamente unánime por el que se reconoce que el éxito académico y laboral no es consecuencia única de un elevado cociente intelectual (Extremera \& Fernández-Berrocal, 2006) y que, además, no se trata de una capacidad inmodificable, sino que puede desarrollarse a través de la instrucción (Gardner, 2001). Es por ello que el rendimiento académico debe ser abordado íntegra y globalmente, incluyendo todas aquellas variables que juegan un papel en su desarrollo óptimo. En este sentido, junto con los aspectos puramente cognitivos, deben aparecer los componentes sociales y emocionales, los cuales no son independientes entre sí. Autores de prestigio en el campo, tales como Gardner (1999), de Maschwitz (2001) o Pérez (2001), entienden así la inteligencia y la definen como la competencia para solucionar problemas del día a día de manera creativa y flexible, suscitar productos nuevos y modificar el conocimiento que se adquiere. Según Montes y Lerner (2011), el rendimiento académico comprende la interrelación entre el proceso de aprendizaje y los resultados tangibles en valores predeterminados, tales como la creatividad y las inteligencias múltiples, incluida la emocional.

Dentro de esta visión holística del rendimiento se enmarca la presente investigación, en la que se consideran los aspectos socioemocionales como precursores del rendimiento integral del individuo y de su desenvolvimiento en la sociedad actual. Compartimos así la idea de que hoy en día hay que formar a alumnos social y emocionalmente inteligentes y, por ende, creativos. Ser inteligente implica, además de serlo cognitivamente, ser creativo y eficaz tanto emocional como socialmente (Sternberg, Grigorenko, \& Bundy, 2001).

\section{Evolución del concepto de inteligencia}

Cada vez está más consensuada la idea de que la inteligencia es un constructo multifactorial que abarca distintos tipos de inteligencias, las cuales aportan al individuo competencias para desenvolverse en todos los ámbitos de su vida, el académico, el laboral, el familiar o el social. En esta línea, Gardner (1983) introduce la teoría de las Inteligencias Múltiples, en la que se considera la inteligencia como un compendio de inteligencias diversas (lingüística, lógico-matemática, espacial, musical, cinestésica, intrapersonal, interpersonal y naturalista). Cada una de ellas se desarrolla en mayor o menor grado en cada individuo en base a sus experiencias y, por ende, a su proceso de enseñanza-aprendizaje. $\mathrm{Si}$ bien es cierto que cada una de estas inteligencias debe ser fomentada desde las aulas en todos los niveles educativos (Amstrong, 2006; Guzmán \& Castro, 2005), y que algunas iniciativas van en esa dirección (Delgado, 2013; Márquez, Guzmán, \& Burgos, 2016; Nadal, 2015), estas se centran en poblaciones y áreas muy concretas estando menos extendidas de lo que sería deseable en la educación actual, donde predominan metodologías más tradicionales (González, 2014).

Diversos trabajos en educación secundaria y universidad, como por ejemplo los realizados por Durán-Aponte, Elvira-Valdés y Pujol (2014), Pérez y Cupani (2008) o Pérez, Cupani y Ayllón (2005), han puesto de manifiesto que los ocho tipos de inteligencia contribuyen a explicar un porcentaje significativo del rendimiento de los estudiantes.

\section{La inteligencia emocional}

El estudio de esta inteligencia se encuentra en pleno auge durante las últimas décadas debido a su repercusión en la vida del individuo, asociada a su manera de pensar y actuar y, por tanto, a su rendimiento (Pérez \& Castejón, 2006; Pulido \& Herrera, 2017). Uno de los autores más reconocidos en este campo es Goleman (1995), que parte de la concepción de la inteligencia emocional como competencia ligada al logro académico y profesional y, por tanto, que favorecerá la adaptación del individuo al medio en el que se desenvuelve (Extremera \& Fernández-Berrocal, 2006). Por su parte, Bisquerra (2011) define esta competencia como la capacidad de tomar conciencia y autorregular, de manera autónoma, las emociones $\mathrm{y}$, dentro de esa conceptualización, destaca como competencias esenciales el conocimiento, la regulación, la autonomía, las habilidades sociales y de vida. 
Diversos estudios (Pérez \& Castejón, 2006) han evidenciado la relación existente entre la inteligencia emocional y el logro académico en estudiantes universitarios y en otros niveles educativos (Sospedra-Baeza, Hidalgo-Fuentes, \& Martínez-Álvarez, 2018; Sospedra-Baeza, Martínez-Álvarez, \& Hidalgo-Fuentes, 2018) Si bien es cierto que existen también algunos estudios que no han reflejado esta relación tan claramente (Barchard, 2003; Bastian, Burns, \& Nettelbeck, 2005).

La educación emocional juega un rol protagonista en el terreno educativo ya desde los niveles iniciales y a lo largo de toda la vida (Bisquerra, 2011). En las últimas décadas, han comenzado a emerger trabajos centrados en el desarrollo de las diferentes habilidades emocionales en diferentes países y niveles educativos (Bisquerra, 2011; Lopes \& Salovey, 2004).

\section{El proceso creativo}

Con la introducción por parte de Piaget del Constructivismo, se comenzó a considerar el proceso creativo como la síntesis de múltiples dimensiones, $\mathrm{y}$, por tanto, como un constructo abstracto y complejo (Esquivias, 2004). A día de hoy, cada vez está más extendida la idea de que la creatividad es un factor esencial en el desarrollo humano, en todos los contextos y a lo largo de todo el ciclo vital, ya que permite a la persona resolver los diversos y complejos problemas con los que se encuentra (Blázquez, 2009). Más concretamente, se puede definir la creatividad como una capacidad a desarrollar y optimizar a través de la instrucción para promover la creación de productos originales y novedosos (Runco \& Jaeger, 2012). Un aspecto clave del pensamiento creativo es que se caracteriza por la originalidad, la expresividad y la flexibilidad mental que supone, permitiendo a la persona encontrar soluciones novedosas y eficaces ante situaciones problemáticas (Pizarro, Bedell, \& Bloom, 2006).

En el contexto educativo más tradicional, la creatividad ha sido una variable poco atendida y estudiada en general (Esquivias, 2004). Pese a ello, autores de prestigio en el campo como Amabile (1983) se han centrado desde hace décadas en otorgarle el papel principal que tiene este proceso, y poco a poco han surgido trabajos focalizados en el análisis y evaluación al hilo de estas investigaciones pioneras. Si bien es cierto que en algunos estudios realizados más recientemente resulta ambigua la relación de la creativi- dad con el rendimiento académico, la tendencia actual (Bermejo, Ruíz, Ferrándiz, Soto, \& Sainz, 2014; Palanniappan, 2007), es que existe una estrecha y directa conexión entre la creatividad y el rendimiento, tanto a nivel global como en diferentes competencias y disciplinas (Bermejo, et al., 2014; $\mathrm{Hu} \&$ Adey, 2002). A nivel de intervención, existen programas diseñados con el fin de mejorar la creatividad en los estudiantes de diferentes niveles educativos, aunque son más frecuentes en los niveles iniciales (Bermejo et al., 2014). Una instrucción estable a lo largo de toda la escolaridad, que promueva de manera eficaz los procesos creativos de originalidad, expresividad y flexibilidad mental ayudan y optimizan el rendimiento, tanto académico como personal, social y laboral, de los estudiantes (Prieto, López, Bermejo, Renzulli, \& Castejón, 2002).

\section{Semejanzas y diferencias entre los aspectos so- cioemocionales}

Las inteligencias, incluida y con un importante peso la emocional, y la creatividad son aspectos esenciales dada la implicación e influencia en todos los ámbitos de la vida. Además, investigaciones llevadas a cabo en diferentes niveles y contextos y con muestras amplias de estudiantes, ponen de manifiesto las conexiones entre dichas variables (Pulido \& Herrera, 2017). De ahí que estos aspectos deban considerarse como partes de un todo fomentando su presencia y desarrollo de manera conjunta con el fin de optimizar el rendimiento y la resolución eficaz de problemas (Fernando, Prieto, Ferrándiz, \& Sánchez, 2005).

De manera paralela al estudio de la relación entre las variables mencionadas, un campo de investigación que está surgiendo en los últimos años es el análisis de la existencia de posibles diferencias en cuanto al desarrollo de las inteligencias múltiples, las competencias emocionales y la creatividad en personas de diferente sexo y edad. Los resultados de los trabajos hasta el momento realizados lanzan resultados diversos y, en ocasiones, contradictorios entre sí. Así, en el ámbito de las inteligencias múltiples se han encontrado diferencias en función del sexo, siendo las mujeres las que obtienen mejor rendimiento en cuanto a las inteligencias musical e interpersonal y los varones en su inteligencia lógico-matemática (Llor et al., 2012). También existen estudios que ponen de manifiesto diferencias, en función del sexo y de la edad, en los estilos cognitivos de per- 
sonas adultas (Muglia, 2009). Adicionalmente, se han encontrado diferencias en inteligencia emocional en función del sexo, siendo las mujeres universitarias las más favorecidas con respecto a sus compañeros masculinos (López-Barajas \& Vallejo, 2015). Respecto a la creatividad, los resultados han sido distintos e incluso contradictorios, Esquivias (1997) con alumnos de Educación Primaria y Elisondo y Donolo (2011) en Universidad, no encontraron diferencias significativas en el nivel de creatividad mostrado por varones y mujeres. Por el contrario, Bermejo y sus colaborados (2014) sí hallaron diferencias en estudiantes universitarios. Concretamente, encontraron que el nivel de creatividad disminuía según iban avanzando en su carrera, lo que podría estar apuntando a un descenso de la creatividad tras el punto cumbre que establecen algunos autores a los 16 años (Smith \& Carlsson, 1990). Por su parte, Chen (2013) mostró en su investigación que los hombres universitarios sobresalían en mayor medida en cuanto a su creatividad que las mujeres, pero no encontró una relación entre el grado de desarrollo de este proceso y el nivel educativo, resultados coherentes con lo encontrado previamente por Elisondo y Donolo (2011). Por tanto, lo aquí presentado, revela la ambigüedad existente en cuanto a la existencia o no de diferencias en inteligencia, competencia emocional y creatividad en función de la edad, nivel educativo y sexo.

Dentro del contexto teórico presentado, se ha puesto de relieve el consenso que paulatinamente, y desde todos los agentes educativos, se va formando en torno a la premisa de que una formación integral requiere, además de un desarrollo intelectual, una intervención dirigida a potenciar las competencias múltiples de la inteligencia, siendo esenciales los aspectos emocionales y creativos, de manera transversal y a lo largo de todo el ciclo vital para dar respuesta a las demandas del cambiante mundo en el que vivimos. Así pues, se plantea el presente estudio para evaluar, relacionar y comparar el grado de autoeficacia percibida por los estudiantes del grado de trabajo social en cuanto a cada una de las inteligencias múltiples, de manera más profunda respecto a la inteligencia emocional, a la creatividad y al rendimiento académico. Para ello, los objetivos específicos que se plantearon fueron:

- Estudiar el grado alcanzado por los estudiantes universitarios en las inteligencias múltiples, inteligencia emocional, creatividad y rendimiento académico.
- Evaluar las diferencias en cuanto a sexo, curso y rendimiento en las variables evaluadas.

- Analizar las correlaciones existentes entre los aspectos valorados en el estudio.

\section{Método}

\section{Participantes}

La muestra está formada por 100 estudiantes del grado de Trabajo Social de la Universitat de València de edades comprendidas entre $\operatorname{los} 17 \mathrm{y}$ los 50 años $(M=20.45 ; D T=4.45)$. Del total de participantes, 91 eran mujeres $(91 \%)$ y 9 varones (9\%). Los alumnos, elegidos en un muestreo por conveniencia, pertenecían el $72 \%$ de ellos a los cursos de primero ( Medad $=19.68 ; D T=4.30)$ y el $28 \%$ a los de tercero $($ Medad $=22.43 ; D T=4.60)$.

\section{Instrumentos}

- Cuestionario autoadministrado creado ad hoc para recoger datos sociodemográficos (edad y sexo) y nota media de bachillerato (medida usada en el estudio como indicador de rendimiento académico).

- Test de Inteligencia Creativa (CREA), una medida cognitiva de la creatividad (Corbalán et al., 2003). Esta prueba tiene como objetivo la evaluación de la creatividad de los sujetos a través de la capacidad de los mismos para elaborar preguntas sobre un dibujo. Cada pregunta supone la activación de un nuevo esquema cognitivo, ofreciendo una medida indirecta de la creatividad de los sujetos (Donolo \& Elisondo, 2007). La prueba cuenta con tres formas existentes con sus correspondientes dibujos: A y B, destinadas a adolescentes y adultos; y $\mathrm{C}$, empleada para niños. En los tres casos, el tiempo de aplicación de la prueba, durante el que los sujetos deben formular de manera escrita tantas preguntas referidas al dibujo correspondiente como puedan, es de cuatro minutos. La puntuación directa es transformada en una puntuación centil que, a su vez, es interpretada como creatividad baja (centiles 1 a 25), media (centiles 26 a 74) o alta (centiles 75 a 99). En este estudio se utilizó la forma B.

- Trait Meta-Mood Scale adaptada. La prueba TMMS-24 (Fernández-Berrocal, Extremera, \& Ramos, 2004) es la versión reducida y adaptada al español de la TMMS-48 (Salovey, Mayer, Goldman, Turvey, \& Palfai, 1995), una escala de inteligencia emocional autopercibida a través de 
las dimensiones de: i) atención a los sentimientos, que representa el grado en que las personas creen prestar atención a sus propios estados emocionales; ii)claridad emocional, definida como la capacidad percibida para identificar y comprender correctamente las propias emociones y, iii) reparación de las emociones, o habilidad subjetiva para eliminar las emociones negativas y prolongar las positivas. La versión española mantiene las tres dimensiones de la escala original pero los ítems se han reducido de cuarenta y ocho a veinticuatro, ocho por cada una de las dimensiones medidas, que se contestan a través de una escala Likert de cinco puntos de anclaje $(1=$ Nada de acuerdo, 5 = Totalmente de acuerdo).

- Inventario de Autoeficacia para Inteligencias Múltiples Revisado (IAMI-R) de Pérez y Cupani (2008) proporciona una medida de autoeficacia en los ocho tipos de inteligencia propuestos por Gardner (1999): lingüística (IL), lógico-matemática (ILM), espacial (IE), cinestésica-corporal (ICC), musical (IM), interpersonal (IInter), intrapersonal (IIntra) y naturalista (IN). La prueba consta de cuarenta y ocho ítems en los que se evalúa de la confianza de los sujetos para realizar correctamente diferentes actividades relacionadas con los ocho factores de inteligencia valorados (seis ítems por cada uno de los factores) a través de una escala Likert de 10 puntos de anclaje $(1=$ no puedo realizar esta actividad, $10=$ muy seguro de poder realizar correctamente esta actividad).

\section{Procedimiento}

Las pruebas se administraron, siguiendo las instrucciones correspondientes de cada una de ellas, dentro del aula en el horario académico de los estudiantes por uno de los autores de la investigación. Todos los alumnos participaron de forma voluntaria después de informarles del alcance y objetivos del estudio.

\section{Análisis de datos}

Los datos fueron introducidos, codificados y depurados con el paquete estadístico SPSS 24.0 para Windows. Se comprobaron los supuestos de normalidad y homocedasticidad y se realizaron análisis estadísticos descriptivos e inferenciales aplicando la prueba paramétrica $t$ de Student y la prueba U de Mann-Whitney para dos muestras independientes en función del sexo, del curso y del rendimiento académico en función del cumplimiento o no de los supuestos de aplicación. Para la variable rendimiento académico se utilizó la nota media de bachillerato de los sujetos, dividiéndoles en dos grupos (rendimiento bajo y alto) en función de si estaban por debajo o por encima del centil 50 de dicha variable.

\section{Resultados}

Los estadísticos descriptivos de cada variable en el total de la muestra, por sexo y curso se presentan en la tabla 1. En las tres dimensiones de la inteligencia emocional autopercibida (TMMS-24) los sujetos de la muestra obtienen puntuaciones adecuadas; por el contrario, la puntuación de creatividad alcanzada es calificada como baja al encontrarse por debajo del centil 25. Los sujetos tienen más confianza en realizar correctamente actividades relacionadas con las inteligencias interpersonal, intrapersonal y lingüística.

En cuanto a las diferencias por sexo, las mujeres presentan un rendimiento académico superior al de los hombres (Hombres $=6.91 ; S D$ $=0.80 ;$ Mujeres $=7.33 ; S D=0.89)$, mientras que la puntuación en la prueba de creatividad es también algo superior en el caso de las mujeres. Por el contrario, los hombres obtienen puntuaciones más altas en los tres componentes de la inteligencia emocional. En cuanto al curso, los alumnos de primero presentan valores superiores en creatividad que los de primero; por el contrario, los alumnos de tercero muestran mayores puntuaciones en los tres componentes de la inteligencia emocional percibida. Las puntuaciones medias obtenidas por los estudiantes de primer y tercer curso en las diferentes inteligencias múltiples son similares en casi todas ellas, obteniéndose las mayores diferencias en las inteligencias lógico-matemática, intrapersonal y naturalista.

Para analizar las diferencias entre los grupos, se comprobó el cumplimiento de los supuestos de homocedasticidad y normalidad. La homocedasticidad mediante la prueba de Levene para la igualdad de varianzas y la normalidad con la prueba de Kolmogorov-Smirnov. En el caso de cumplimiento de los supuestos se aplicó la prueba $\mathrm{t}$ de Student para dos muestras independientes, mientras que en el caso de incumplimiento de los supuestos se aplicó la alternativa no paramétrica U de Mann-Whitney. 
Tabla 1

Estadísticos descriptivos por sexo, curso y total

\begin{tabular}{|c|c|c|c|c|c|c|}
\hline & Sexo & $M$ & $S D$ & Curso & $M$ & $S D$ \\
\hline \multirow[t]{3}{*}{ Edad } & Hombre & 19.00 & 1.41 & Primero & 19.68 & 4.30 \\
\hline & Mujer & 20.59 & 4.71 & Tercero & 22.43 & 4.60 \\
\hline & Total & 20.45 & 4.54 & & & \\
\hline \multirow{3}{*}{$\begin{array}{l}\text { Rendimiento } \\
\text { académico }\end{array}$} & Hombre & 6.91 & 0.80 & Primero & 7.36 & 0.89 \\
\hline & Mujer & 7.33 & 0.89 & Tercero & 7.05 & 0.86 \\
\hline & Total & 7.27 & 0.89 & & & \\
\hline \multirow[t]{3}{*}{ CREA } & Hombre & 20.22 & 15.21 & Primero & 23.56 & 17.24 \\
\hline & Mujer & 23.42 & 17.30 & Tercero & 22.04 & 16.98 \\
\hline & Total & 23.13 & 17.10 & & & \\
\hline \multirow{3}{*}{$\begin{array}{l}\text { TMMS } \\
\text { atención }\end{array}$} & Hombre & 29.56 & 5.88 & Primero & 27.40 & 6.70 \\
\hline & Mujer & 27.41 & 6.88 & Tercero & 28.11 & 7.17 \\
\hline & Total & 27.60 & 6.80 & & & \\
\hline \multirow{3}{*}{$\begin{array}{l}\text { TMMS } \\
\text { claridad }\end{array}$} & Hombre & 27.11 & 6.49 & Primero & 24.35 & 6.58 \\
\hline & Mujer & 24.76 & 6.38 & Tercero & 26.57 & 5.65 \\
\hline & Total & 27.97 & 6.39 & & & \\
\hline \multirow{3}{*}{$\begin{array}{l}\text { TMMS } \\
\text { reparación }\end{array}$} & Hombre & 26.00 & 9.06 & Primero & 24.68 & 6.37 \\
\hline & Mujer & 24.98 & 6.04 & Tercero & 26.07 & 6.18 \\
\hline & Total & 25.07 & 6.31 & & & \\
\hline \multirow[t]{3}{*}{ I. Lingüística } & Hombre & 7.57 & 1.73 & Primero & 6.87 & 1.91 \\
\hline & Mujer & 6.82 & 1.94 & Tercero & 6.93 & 2.01 \\
\hline & Total & 6.88 & 1.93 & & & \\
\hline \multirow{3}{*}{$\begin{array}{l}\text { I. Lógico- } \\
\text { matemática }\end{array}$} & Hombre & 5.30 & 2.96 & Primero & 4.48 & 2.29 \\
\hline & Mujer & 4.09 & 2.21 & Tercero & 3.48 & 2.19 \\
\hline & Total & 4.20 & 2.30 & & & \\
\hline \multirow[t]{3}{*}{ I. Espacial } & Hombre & 4.65 & 2.61 & Primero & 4.07 & 2.43 \\
\hline & Mujer & 3.91 & 2.39 & Tercero & 3.76 & 2.38 \\
\hline & Total & 3.98 & 2.41 & & & \\
\hline \multirow[t]{3}{*}{ I. Musical } & Hombre & 3.19 & 2.49 & Primero & 3.88 & 2.69 \\
\hline & Mujer & 3.98 & 2.71 & Tercero & 3.99 & 2.73 \\
\hline & Total & 3.91 & 2.69 & & & \\
\hline \multirow[t]{3}{*}{ I. Interpersonal } & Hombre & 8.30 & 1.26 & Primero & 7.78 & 1.20 \\
\hline & Mujer & 7.73 & 1.14 & Tercero & 7.79 & 1.06 \\
\hline & Total & 7.78 & 1.16 & & & \\
\hline \multirow{3}{*}{$\begin{array}{l}\text { I. Cinestésica- } \\
\text { corporal }\end{array}$} & Hombre & 4.78 & 3.06 & Primero & 5.43 & 2.06 \\
\hline & Mujer & 5.49 & 1.99 & Tercero & 5.41 & 2.22 \\
\hline & Total & 5.42 & 2.10 & & & \\
\hline \multirow{3}{*}{ I. Intrapersonal } & Hombre & 7.43 & 1.34 & Primero & 6.74 & 1.59 \\
\hline & Mujer & 6.92 & 1.57 & Tercero & 7.57 & 1.30 \\
\hline & Total & 6.97 & 1.55 & & & \\
\hline \multirow[t]{3}{*}{ I. Naturalista } & Hombre & 5.54 & 2.88 & Primero & 4.39 & 1.95 \\
\hline & Mujer & 4.09 & 1.74 & Tercero & 3.78 & 1.71 \\
\hline & Total & 4.22 & 1.89 & & & \\
\hline
\end{tabular}

Tabla 2

Resultados prueba t de Student en función del sexo

\begin{tabular}{llllll}
\hline & $t$ & $g l$ & $p$ & \multicolumn{2}{c}{ IC $95 \%$} \\
\hline TMMS claridad & 1.05 & 98 & 0.294 & -2.07 & 6.78 \\
I. Lógico- & 1.51 & 98 & 0.135 & -0.38 & 2.79 \\
matemática & 0.93 & 98 & 0.356 & -0.57 & 1.58 \\
I. Intrapersonal & 1.48 & 8.59 & 0.174 & -0.78 & 3.68 \\
I. Naturalista & & & & &
\end{tabular}

No existen diferencias en forma estadísticamente significativas (ver tabla 2 y tabla 3 ) entre hombres y mujeres en ninguna de las variables estudiadas.

Se han obtenido diferencias en forma estadísticamente significativas entre los cursos de primero $\mathrm{y}$ tercero en inteligencia intrapersonal $t(98)=-2.46$, $p=0.016$, obteniéndose una diferencia a favor de los alumnos de tercero entre 1.5 y 0.16 puntos, con un nivel de confianza del 95\% (ver tabla 4); $\mathrm{y}$ en inteligencia lógico-matemática (ver tabla 5) $Z=-2.01, p=0.045$ (ver tabla 5), siendo mayor la autoeficacia percibida en este tipo de inteligencia en los alumnos de primero.

Se han obtenido diferencias en forma estadísticamente significativas en función del rendimiento académico de los alumnos (ver tabla 6 y tabla 7) en inteligencia lógico-matemática $t(64)=-2.00$, $p=0.050$ con un nivel de confianza del $95 \%$, siendo mayor la autoeficacia percibida en el caso de los alumnos con un rendimiento académico alto.

El rendimiento académico se relaciona positiva y en forma estadísticamente significativa (ver tabla 8) con la inteligencia cinestésica-corporal.

Los componentes de atención a los sentimientos y claridad emocional de la escala TMMS24 se relacionan entre ellos. Los componentes atención a los sentimientos y claridad emocional se relacionan con las inteligencias intrapersonal e interpersonal, mientras que la reparación de las emociones muestra relación con la inteligencia intrapersonal. El componente claridad emocional tiene relación con todas las inteligencias múltiples, a excepción de la espacial y la musical. El componente de reparación presenta relación con todas las inteligencias salvo con la lógicomatemática. La creatividad se relaciona con las inteligencias espacial e interpersonal. 
Tabla 3

Resultados prueba $U$ de Mann-Whitney en función del sexo

\begin{tabular}{lccccccccc}
\hline & $\begin{array}{c}\text { Rendimiento } \\
\text { académico }\end{array}$ & $\begin{array}{c}\text { TMMS } \\
\text { Atención }\end{array}$ & $\begin{array}{c}\text { TMMS } \\
\text { Reparación }\end{array}$ & $\begin{array}{c}\text { I. } \\
\text { Lingǘstica }\end{array}$ & I. Espacial & I. Musical & $\begin{array}{c}\text { I. } \\
\text { Interpersonal }\end{array}$ & $\begin{array}{c}\text { I. } \\
\text { Cinestésica- } \\
\text { corporal }\end{array}$ & CREA \\
\hline $\begin{array}{l}\text { U de Mann- } \\
\text { Whitney }\end{array}$ & 192.5 & 336.50 & 399.00 & 323.50 & 334.50 & 313.00 & 297.50 & 370.50 & 362.00 \\
$\begin{array}{l}\text { Z } \\
\begin{array}{l}\text { Sig. asintótica } \\
\text { (bilateral) }\end{array}\end{array}$ & -1.2 & -0.88 & -0.13 & -1.04 & -0.90 & -1.16 & -1.35 & -0.47 & -0.58 \\
\hline
\end{tabular}

Tabla 4

Resultados prueba t de Student en función del curso

\begin{tabular}{llllll}
\hline & $t$ & $g l$ & $p$ & \multicolumn{1}{l}{ IC 95\% } \\
\hline Rendimiento & 1.28 & 64 & 0.205 & -0.17 & 0.79 \\
académico & -0.46 & 98 & 0.644 & -3.72 & 2.31 \\
TMMS atención & -1.57 & 98 & 0.119 & -5.03 & 0.58 \\
TMMS claridad & -2.46 & 98 & 0.016 & -1.50 & -0.16 \\
\hline
\end{tabular}

Tabla 5

Resultados prueba U de Mann-Whitney en función del curso

\begin{tabular}{|c|c|c|c|c|c|c|c|c|c|}
\hline & $\begin{array}{c}\text { TMMS } \\
\text { Reparación }\end{array}$ & $\begin{array}{c}\text { I. } \\
\text { Lingüística }\end{array}$ & $\begin{array}{l}\text { I. Lógico } \\
\text { matemática }\end{array}$ & $\begin{array}{c}\text { I. } \\
\text { Espacial }\end{array}$ & $\begin{array}{c}\text { I. } \\
\text { Musical }\end{array}$ & $\begin{array}{c}\text { I. } \\
\text { Interpersonal }\end{array}$ & $\begin{array}{l}\text { I. } \\
\text { Cinestésica- } \\
\text { corporal }\end{array}$ & $\begin{array}{c}\text { I. } \\
\text { Naturalista }\end{array}$ & CREA \\
\hline $\begin{array}{l}\text { Ude Mann- } \\
\text { Whitney }\end{array}$ & 874.00 & 981.50 & 746.50 & 951.50 & 991.00 & 994.00 & 1000.50 & 845.50 & 960.50 \\
\hline Z & -1.03 & -0.20 & -2.01 & -0.43 & -0.13 & -0.11 & -0.06 & -1.25 & -0.37 \\
\hline $\begin{array}{l}\text { Sig. asintotica } \\
\text { (bilateral) }\end{array}$ & 0.303 & 0.839 & 0.045 & 0.664 & 0.896 & 0.914 & 0.954 & 0.212 & 0.713 \\
\hline
\end{tabular}

Tabla 6

Resultados prueba t de Student en función del rendimiento académico (bajo/alto)

\begin{tabular}{lllllc}
\hline & $t$ & $g l$ & $p$ & \multicolumn{2}{c}{ IC 95\% } \\
\hline TMMS atención & -0.28 & 64 & 0.778 & -3.65 & 2.75 \\
TMMS claridad & -0.67 & 55.68 & 0.508 & -4.00 & 2.00 \\
I. Lógico-matemática & -2.00 & 64 & 0.050 & -2.13 & -0.00 \\
I. Intrapersonal & 0.53 & 64 & 0.595 & -0.56 & 0.98 \\
I. Naturalista & -1.08 & 64 & 0.285 & -1.54 & 0.46 \\
\hline
\end{tabular}

Tabla 7

Resultados prueba U de Mann-Whitney en función del rendimiento académico (bajo/alto)

\begin{tabular}{lccccccc}
\hline & $\begin{array}{c}\text { TMMS } \\
\text { reparación }\end{array}$ & I. Lingüística & I. Espacial & I. Musical & I. Interpersonal & $\begin{array}{c}\text { I. Cinestésica- } \\
\text { corporal }\end{array}$ & CREA \\
\hline Ude Mann- & 1064.00 & 1042.50 & 1019.50 & 930.50 & 972.50 & 1019.00 & 973.00 \\
Whitney & -0.30 & -0.46 & -0.63 & -1.28 & -0.98 & -0.63 & -0.98 \\
$\begin{array}{l}\text { Z } \\
\begin{array}{l}\text { Sig. asintótica } \\
\text { (bilateral) }\end{array}\end{array}$ & 0.761 & 0.644 & 0.528 & 0.199 & 0.329 & 0.526 & 0.327 \\
\hline
\end{tabular}


Tabla 8

Correlaciones bivariadas entre el rendimiento, creatividad, las dimensiones de la Inteligencia Emocional y las Inteligencias Múltiples

\begin{tabular}{|c|c|c|c|c|c|c|c|c|c|c|c|c|c|}
\hline & 1 & 2 & 3 & 4 & 5 & 6 & 7 & 8 & 9 & 10 & 11 & 12 & 13 \\
\hline 1. Rendimiento académico & 1 & & & & & & & & & & & & \\
\hline 2. CREA & .15 & 1 & & & & & & & & & & & \\
\hline 3. TMMS atención & .01 & -.17 & 1 & & & & & & & & & & \\
\hline 4. TMMS claridad & .02 & -.02 & $.28 * *$ & 1 & & & & & & & & & \\
\hline 5. TMMS reparación & -.01 & .14 & -.03 & .18 & 1 & & & & & & & & \\
\hline 6. I. Lingüística & .22 & .13 & .20 & $.24 *$ & .02 & 1 & & & & & & & \\
\hline 7. I. Lógico-matemática & .22 & .07 & $.21^{*}$ & .09 & .01 & $.29^{*}$ & 1 & & & & & & \\
\hline 8. I. Espacial & .22 & .17 & .07 & .13 & .11 & .18 & $.30 * *$ & 1 & & & & & \\
\hline 9. I. Musical & .22 & .11 & .14 & $.22 *$ & -.06 & .14 & $.23^{*}$ & .17 & 1 & & & & \\
\hline 10. I. Interpersonal & .07 & .04 & $.22 *$ & $.46^{* *}$ & .01 & $.40 * *$ & $.20 *$ & .04 & .12 & 1 & & & \\
\hline 11. I. Cinestésica-corporal & $.26^{*}$ & -.06 & .07 & .09 & $.22 *$ & .07 & $.29 * *$ & $.31 * *$ & .17 & .13 & 1 & & \\
\hline 12. I. Intrapersonal & -.09 & -.01 & $.25^{*}$ & $.59 * *$ & $.24 *$ & $.32 * *$ & -.01 & .04 & -.01 & $.34 * *$ & .11 & 1 & \\
\hline 13. Naturalista & .22 & .09 & .08 & .061 & .07 & $.25^{*}$ & $.24 *$ & $.32 * *$ & $.24 *$ & .12 & $.45^{* *}$ & $.22 *$ & 1 \\
\hline
\end{tabular}

${ }^{*} p<.05,{ }^{*} p<.01$

\section{Discusión y conclusiones}

Como fin principal de la investigación descrita previamente nos habíamos propuesto evaluar, relacionar y comparar el grado de autoeficacia percibida por los estudiantes a nivel de grado universitario de trabajo social en cuanto a cada una de las inteligencias múltiples, de manera más concreta respecto a la inteligencia emocional, a la creatividad y al rendimiento académico.

Dentro de nuestro objetivo global, nos propusimos indagar acerca de cuestiones consideradas esenciales para avanzar en el campo de las inteligencias y la creatividad en relación al rendimiento de los estudiantes universitarios $\mathrm{y}$, por ello, hemos investigado tres aspectos de los que se han obtenido resultados interesantes y novedosos, lo cual supone un aporte que puede ser de interés para otros estudios y para la propia planificación académico-curricular.

A nivel descriptivo, hemos estudiado el nivel que tienen los estudiantes de trabajo social en las inteligencias múltiples, inteligencia emocional, creatividad y rendimiento académico. A partir de ello, y en coherencia con algunas investigaciones previas (Martínez, Llamas-Salgado, \& LópezFernández, 2016), los estudiantes de este nivel educativo presentan niveles medios en cuanto a sus inteligencias múltiples, incluida la emocional y de medio a alto en rendimiento. No sucede lo mismo respecto a la creatividad, en cuanto a la que hemos encontrado que los estudiantes de la muestra puntuaban bajo, a diferencia de lo hallado en recientes investigaciones, como la llevada a cabo por Chiecher, Elisondo, Paoloni y Donolo (2018) con estudiantes de ingeniería que presentaban un nivel medio de creatividad o la realizada por Martínez et al. (2016) en la que estudiantes del área gráfica mostraban niveles altos de esta variable. Este dato no llama la atención teniendo en consideración que los niveles de estas variables parecen relacionarse con la elección de carrera que hacen los estudiantes (Pérez \& Cupani, 2008).

El siguiente paso que dimos en esta investigación fue el estudio de las diferencias existentes en función del sexo, el curso académico $y$ el rendimiento, lo que nos ha aportado información relevante $\mathrm{y}$, en parte, coherente con lo indicado por otros autores previos. Así, no hallamos diferencias entre sexos y niveles de rendimiento en ninguna de las variables analizadas, a diferencia de algunos estudios previos como el realizado por Llor et al. (2012). A pesar de que los datos de esta investigación deben tomarse con cierta cautela en relación a las diferencias entre hombres y mujeres, dado que 
el porcentaje de participantes femeninas fue más elevado que el de hombres, se observa, como se consideraba hasta el momento, que los datos son en ocasiones contradictorios con respecto a las diferencias en función del sexo en las variables sociemocionales y, por tanto, se debe mantener el interés por indagar y clarificar este tema en auge en los últimos años.

Con respecto al nivel educativo de los estudiantes, encontramos que los que cursaban tercero de trabajo social presentaron niveles más elevados de inteligencia intrapersonal, lo que haría pensar que aumenta con la edad y con las competencias que adquieren durante los primeros cursos del grado universitario; pero puntuaron más bajo que sus compañeros del primer curso en la inteligencia lógico-matemática, lo cual podría deberse, al menos en parte, a la escasez de asignaturas relacionadas con este ámbito que se imparten los cursos del grado de trabajo social incluidos en el estudio. Como ya pusimos de manifiesto en la introducción teórica de este manuscrito, los datos encontrados por autores previos son ambiguos a este respecto por lo que, los hallazgos de este estudio nos ofrecen algunas orientaciones acerca de la situación que se encuentran en la línea de trabajos como el de Muglia (2009), en el que hallaba diferencias en cuanto al estilo cognitivo, o de Chen (2013), que no encontró, al igual que en el presente estudio, diferencias en función de la creatividad. $\mathrm{La}$ contribución de las inteligencias múltiples al rendimiento académico ya se había corroborado en estudios previos realizados (Durán-Aponte et al., 2014; Pérez \& Cupani, 2008; Pérez et al., 2005).

El último objetivo perseguido era analizar las correlaciones entre los aspectos valorados en el estudio $\mathrm{y}$, respecto a ello, encontramos diferentes e interesantes datos que ofrecen aportaciones novedosas. Por un lado, el rendimiento se relaciona con la inteligencia cinestésica, un dato concreto poco conocido hasta el momento. En nuestro trabajo encontramos además que los diferentes componentes de la inteligencia emocional analizados se relacionan con inteligencias múltiples. En concreto, el grado en el que los estudiantes consideran que prestan atención a sus emociones se relaciona con su inteligencia lógico-matemática y con la inteligencia emocional, tanto intra como interpersonal. En cuanto a la percepción de su capacidad para identificar y comprender las emociones, esta se encuentra conectada con su inteligencia lingüística, la emocional y también la musical. Por último, la capacidad de regulación y reparación de las emociones positivas y negativas se relaciona con la inteligencia musical y la intrapersonal. En coherencia con lo ya encontrado por investigadores en este campo, las variables sociemocionales analizadas en este estudio no deben considerarse de manera aislada sino como componentes esenciales e interrelacionados en la resolución eficaz de problemas (Fernando et al., 2005).

Gracias a la realización de este estudio hemos sido conscientes de algunas limitaciones que deben ser solventadas en un futuro próximo. Así, por un lado, el tamaño y homogeneidad de la muestra, no han permitido en su totalidad, indagar y analizar claramente las relaciones entre los aspectos incluidos, así como las diferencias entre hombres y mujeres y estudiantes de diferente nivel, por lo que sería interesante continuar avanzando en esta línea y aumentar tanto el número como la variedad de carreras estudiadas de los participantes en futuros estudios para dar respuesta a esta ambigua cuestión.

En síntesis, se concluye a partir de lo discutido que resulta relevante analizar las variables socioemocionales que se han estudiado en la presente investigación con un doble fin, por un lado, clarificar cuáles, de qué modo y en qué momento intervienen cada una de ellas $\mathrm{y}$, por el otro, para poder diseñar programas de intervención centrados en la optimización del rendimiento global de los estudiantes universitarios.

\section{Referencias}

Amabile, T. M. (1983). The Social Psycholoy of Creativity: A Componential Conceptualization. Journal of Personality and Social Psychology, 45 (2), 357-376. doi:10.1037/0022-3514.45.2.357

Amstrong, T. (2006). Las inteligencias múltiples en el aula. Madrid: Paidós.

Barchard, K. A. (2003). Does emotional intelligence assist in the prediction of academic success?. Educational and psychological measurement, 63(5), 840-858. doi:10.1177/0013164403251333

Bastian, V. A., Burns, N. R., \& Nettelbeck, T. (2005). Emotional intelligence predicts life skills, but not as well as personality and cognitive abilities. Personality and individual differences, 39(6), 1135-1145. doi:10.1016/j. paid.2005.04.006 
Bermejo, R., Ruiz, M. J., Ferrándiz, C., Soto, G., \& Sainz, M. (2014). Pensamiento científico-creativo y rendimiento académico. Scientific-creative thinking and academic achievement. Revista de Estudios e Investigación en Psicología y Educación, 1(1), 64-72. doi: 10.17979/ reipe.2014.1.1.24

Bisquerra, R. (Coord.) (2011). Educación emocional. Propuestas para educadores y familias. Bilbao: Desclée de Brower.

Blázquez, A. (2009). Educación y creatividad. Innovación y experiencias educativas. Art. 38. Granada: CSI-F Enseñanza Andalucía.

Corbalán, F. J., Martínez, F., Donolo, D., Alonso, C., Tejerina, M., \& Limiñana, R. M. (2003). CREA. Inteligencia creativa. Una medida cognitiva de la creatividad. Madrid: TEA Ediciones.

Chen Tsai, K. (2013). Examining gender differences in creativity, The International Journal of Social Sciences, 13(1), 115-122.

Chiecher, A. C., Elisondo, R. C., Paoloni, P. V., \& Donolo, D. S. (2018). Creatividad, género y rendimiento académico en ingresantes de ingeniería. Revista Iberoamericana de Educación Superior, 9(24), 138151.

Delgado, M. P. (2013). Aplicación didáctica de las Inteligencias Múltiples. E-motion. Revista de Educación, Motricidad e Investigación, 1, 103-116.

Donolo, D. \& Elisondo, R. (2007). Creatividad para todos. Consideraciones para un grupo particular. Anales de Psicología, 23(1), 147-151.

Durán-Aponte, E., Elvira-Valdés, M., \& Pujol, L. (2014). Validación del Inventario de Autoeficacia para Inteligencias Múltiples Revisado (IAMI-R) en una muestra de universitarios venezolanos, Actualidades Investigativas en Educación, 14(2), 1-23.

Elisondo, R., \& Donolo, D. (2011). Los estímulos en un test de creatividad. Incidencias según género, edad y escolaridad. Boletín de Psicología, 101, 51-65.

Esquivias, M. T. (1997). Estudio evaluativo de tres aproximaciones pedagógicas: ecléctica, Montessori y Freinet, sobre la ejecución de solución de problemas y creatividad, con niños de escuela primaria. (Tesis de Licenciatura). Facultad de Psicología. UNAM. México.

Esquivias, M.T. (2004). Creatividad: Definiciones, antecedentes y aportaciones, Revista Digital Universitaria, 5(1), 1-17.

Extremera, N., \& Fernández-Berrocal, P. (2006). Emotional Intelligence as predictor of mental, social and Physical health in university students. The Spanish Journal of Psychology, 1, 45-51. doi:10.1017/s1138741600005965

Fernández-Berrocal, P., Extremera, N., \& Ramos, N. (2004). Validity and reliability of the Spanish modified version of the Trait Meta-Mood Scale. Psychological reports, 94(3), 751-755. doi:10.2466/pr0.94.3.751-755

Fernando, M., Prieto, M.D., Ferrándiz, C., \& Sánchez, C. (2005). Inteligencia y creatividad. Revista Electrónica de Investigación Psicoeducativa, 3(3), 21-50.

Gardner, H. (1983). Frames of mind. London: Fontana (Trad. Cast., Inteligencias múltiples. Barcelona: Paidós, 1995).

Gardner, H. (1999). Intelligence reframed. Multiple intelligences for the $21^{\text {st }}$ Century. Nueva York: Basic Books.

Gardner, H. S. B. (2001). La inteligencia reformulada: las inteligencias múltiples en el siglo XXI. Barcelona: Paidós.
Goleman, D. P. (1995). Emotional intelligence: Why it can matter more than IQ for character, health and lifelong achievement. Nueva York: Bantam Books.

González, D. (2014). Inteligencias múltiples y dificultades del aprendizaje. Padres y Maestros, 357, 10-14.

Guzmán, B., \& Castro, S. (2005). Las inteligencias múltiples en el aula de clases. Revista de investigación, 58, $177-$ 202.

Hu, W., \& Adey, P. A. (2002). Scientific creativity test for secondary school students. International Journal of Science Education, 24(4), 389-403. doi: 10.1080/09500690110098912.

Llor, L., Ferrando, M., Ferrándiz, C., Hernández, D., Sáinz, M., Prieto, M. D. \& Fernández, M. C. (2012). Inteligencias múltiples y alta habilidad. Aula abierta, 40(1), 27-38

Lopes, P. N., \& Salovey, P. (2004). Toward a broader education: Social, emotional, and practical skills. En H. J. Walberg, M. C. Wang, R. P. Weissberg \& Zins (Eds.): Building School Success on Social and Emotional Learning: What does the research say (pp. 76-93). New York: Teachers College Press.

López-Barajas, D. M., \& Vallejo, A. P. (2015). Diferencias de género en inteligencia emocional percibida en docentes de infantil y primaria. En Aidipe (Ed.), Investigar con y para la sociedad (Vol.1, pp. 369378). Cádiz, España: Bubok. Recuperado de: http:// aidipe2015.aidipe.org.

Martín, E., \& Moreno, A. (2008). Competencia para aprender a aprender. Madrid: Alianza.

Martínez, C. D., Llamas-Salguero, F., \& López-Fernández, V. (2016). Relación entre creatividad, inteligencias múltiples y rendimiento académico en alumnos de enseñanza media técnico profesional del área gráfica. Programa de intervención neuropsicológico utilizando las TIC. Academia y Virtualidad, 9(2), 41-58.

Márquez, M. G., Guzmán, L. R., \& Burgos, B. M. (2016). Mediaciones pedagógicas para el desarrollo de las Inteligencias Múltiples en Educación Superior. Revista Pertinencia Académica, 1, 37-52.

Maschwitz de, E. (2001) Inteligencias Múltiples en la educación de las personas. Disponible en www. godspell.org.ar/inteligencias.htm

Montes, I., \& Lerner, J. (2011). Rendimiento académico de los estudiantes de pregrado de la Universidad EAFIT. Colombia: Universidad de EAFIT.

Muglia Wechsler, S. (2009). Age and gender impact on thinking and creating styles. European journal of Education and Psychology, 2(1), 37-48.

Nadal Vivas, B. (2015). Las inteligencias múltiples como una estrategia didáctica para atender a la diversidad y aprovechar el potencial de todos los alumnos. Revista Nacional e Internacional de Educación Inclusiva, 8(3), 121-136.

Palaniappan, A. K. (2007). Academic Achievement of Groups Formed Based on Creativity and Intelligence. Reviewed Research Papers selected for publication and presentation at the 13th International Conference on Thinking, Norrkoping, Sweden. 1650-3686.

Pérez, E. (2001). Construcción de un Inventario de Autoeficacia para Inteligencias Múltiples. Tesis Doctoral inédita. Facultad de Psicología. Universidad Nacional de Córdoba. Córdoba. Argentina. Disponible en http://www.datavoc.com/orientadores/editoriales/ notas $/$ ?idnota $=147$ 
Pérez, N., \& Castejón, J. L. (2006). La inteligencia emocional como predictor del rendimiento académico en estudiantes universitarios. Ansiedad y Estrés, 12(23), 393-400.

Pérez, E. \& Cupani, M. (2008). Validación del Inventario de Autoeficacia para Inteligencias Múltiples Revisado (IAMI-R). Revista Latinoamericana de Psicología, 40(1), 47-58.

Pérez, E., Cupani, M., \& Ayllon, S. (2005). Predictores de rendimiento académico en la escuela media: habilidades, autoeficacia y rasgos de personalidad. Avaliaçao Psicológica, 4(1), 1-11.

Pizarro, D.A., Detweiler-Bedell, B., \& Bloom, P. (2006). The Creativity of Everyday Moral Reasoning: Empathy, Disgust and Moral Persuasion. In J. C. Kaufman \& J. Baer (Eds.), Creativity and Reason in Cognitive Development. Cambridge University Press. doi:10.1017/ cbo9780511606915.006

Prieto, M. D., López, O., Bermejo , M. R. , Renzulli, J., \& Castejón, J. L. (2002). Evaluación de un programa de desarrollo de la creatividad. Psicothema, 14, 410-414.

Pulido Acosta, F. y Herrera Clavero, F. (2017). La influencia de las emociones sobre el rendimiento académico. Ciencias Psicológicas, 11(1), 29-39. doi: https://doi. org/10.22235/cp.v11i2.1344

Runco, M. A., \& Jaeger, G. J. (2012). The standard definition of creativity. Creativity Research Journal, 24(1), 92-96. doi:10.1080/10400419.2012.650092
Salovey, P., Mayer, J. D., Goldman, S. L., Turvey, C., \& Palfai, T. F. (1995). Emotional attention, clarity, and repair: Exploring emotional intelligence using the Trait Meta-Mood Scale. En J. W. Pennebaker (Ed.), Emotion, disclosure, and health (pp. 125-154). Washington, DC: American Psychological Association. doi:10.1037/10182-006

Smith, G.J., \& Carlsson, I. M. (1990). The creative process: A functional model based on empirical studies from early childhood to middle age. Madison, CT: International Universities Press.

Sospedra-Baeza, M.J., Hidalgo-Fuentes, S.\& MartínezÁlvarez, I (2018a). Inteligencia emocional percibida y rendimiento académico en estudiantes de psicología. En AEPC (Ed.), 6th International Congress of Educational Sciences and Development (p. 1102). Sétubal, Portugal.

Sospedra-Baeza, M.J., Martínez-Álvarez, I. \& HidalgoFuentes, S. (2018b). Inteligencia emocional percibida y rendimiento académico en estudiantes de trabajo social. En AEPC (Ed.), 6th International Congress of Educational Sciences and Development (p. 1103). Sétubal, Portugal.

Sternberg, R. J., Grigorenko, E., \& Bundy, D. A. (2001). The predictive value of IQ. Merrill-Palmer Quarterly, 47(1), 1-41. doi:10.1353/mpq.2001.0005 